\title{
Field-Cycling NMR Relaxation Spectroscopy of Poly(di- $n$-alkylsiloxanes) in Solid, Mesomorphic Liquid, and Isotropic Liquid Phases
}

\author{
R. Kimmich, ,† S. Stapf, ${ }^{\dagger}$ M. Möller, ${ }^{\ddagger}$ R. Out, ${ }^{\ddagger}$ and R.-O. Seitter ${ }^{\dagger}$ \\ Sektion Kernresonanzspektroskopie and Abteilung Organische Chemie III, Universităt Ulm, \\ 89069 Ulm, Germany
}

Received September 28, 1993; Revised Manuscript Received December 14, $1993^{\circ}$

\begin{abstract}
The frequency dependence of the proton spin-lattice relaxation times $T_{1}$ and $T_{10}$ in the laboratory and rotating frames, respectively, is reported for solid and liquid phases of poly(diethylsiloxane) (PDES) and in melts of poly (dimethylsiloxane) (PDMS). The total frequency range is $5 \times 10^{2}-3 \times 10^{8} \mathrm{~Hz}$ and is mainly covered by field-cycling NMR relaxation spectroscopy. The relaxation behavior of PDES in the liquid but ordered mesophase is compared to that of isotropic melts of PDES and PDMS and also to that of nematic main-chain liquid-crystal polymers. The frequency dependences of PDES and PDMS liquids can be represented at low and high frequencies by power laws, section by section. The relaxation behavior in the isotropic melts is entirely equivalent to that previously reported for other polymer species. In the PDES mesophase, the exponents of the power laws are significantly larger and the crossover frequency between the two regimes is reduced. The dynamics in this phase are discussed with respect to the influence of chain modes and order director fluctuations. The main conclusion is, on the whole, that data of the liquid phases are determined by chain modes rather than by local segment fluctuations. The chain dynamics in the PDES mesophase resemble the chain modes in isotropic melts modified for a microstructure with reduced randomness, whereas the influence of order director fluctuations can neither be confirmed nor ruled out.
\end{abstract}

\section{Introduction}

Linear poly(diethylsiloxane) (PDES) and its higher homologues 1,2 form liquid but ordered mesophases in spite of the high chain flexibility and the entire lack of any mesogenic groups. This is in contrast to conventional main- or side-chain liquid-crystalline polymers. The physics of these two types of liquid-crystalline states is therefore expected to be of a different nature.

Dynamic chain modes, whether collective or not, are closely related to the microstructure. Accordingly one is interested in any peculiarities of polymer dynamics in such phases. The purpose of this study therefore is to compare the features of chain dynamics in the PDES mesophase with the behavior of conventional nematic main-chain liquid-crystal polymers and also of isotropic melts.

A powerful technique for such studies is provided by field-cycling NMR relaxation spectroscopy ${ }^{3,4}$ supplemented by conventional measurements of the spin-lattice relaxation times in the laboratory and rotating frames of reference, $T_{1}$ and $T_{1 \rho}$, respectively. The accessible frequency scale for proton resonance ranges from $5 \times 10^{2}$ up to $3 \times 10^{8} \mathrm{~Hz}$, so that the fluctuation rates of the characteristic chain modes can be expected to be covered in the temperature intervals of the phases.

The technique has already been applied extensively to isotropic polymer melts ${ }^{6,6}$ and to main-chain liquid-crystal polymers ${ }^{7}$ apart from studies of monomeric liquid crystals. 8,9 The frequency dependence ("dispersion") of the spin-lattice relaxation time in these systems, $T_{1}=T_{1}(\nu)$, shows features characterizing the molecular dynamics in a rather pronounced and direct way. The dispersion curves can often be represented by power laws which may have theoretical counterparts.

With nematic liquid crystals, for instance, reorientations

† Sektion Kernresonanzspektroskopie.

t Abteilung Organische Chemie III.

- Abstract published in Advance ACS Abstracts, February 15, 1994.

$0024-9297 / 94 / 2227-1505 \$ 04.50 / 0$ by order fluctuations are characterized by the proportionality

$$
T_{1} \propto \nu^{1 / 2}
$$

i.e., by a power law which should dominate the $T_{1}$ dispersion at low frequencies. ${ }^{10-16}$ This prediction was found to be compatible with experimental data of nematic liquid-crystal polymers. ${ }^{7}$

$T_{1}$ dispersion experiments with isotropic polymer melts, on the other hand, show a whole set of power-law regimes typically given by

$$
T_{1} \propto \nu^{x} \quad \text { where } x=0.45,0.25,0.5
$$

It was shown that these findings can be interpreted in a rather detailed manner by limiting cases of certain polymer theories. $5,6,17$

In the following we present $T_{1}$ dispersion data of PDES in solid, mesomorphic, and isotropic liquid phases. For comparison, isotropic poly(dimethylsiloxane) (PDMS) melts have also been studied as a material without any liquid mesophases at normal pressure. The experimental results are discussed in the context of the background outlined above.

\section{Experimental Section}

A home-built field-cycling NMR relaxation spectrometer with a liquid-nitrogen-cooled copper magnet was used for the proton frequency range up to $2 \times 10^{7} \mathrm{~Hz}$. Spin-lattice relaxation times at higher frequencies and spin-lattice relaxation times in the rotating frame were recorded using conventional techniques on Bruker SXP 4-100 and MSL 300 spectrometers operating at 90 and $300 \mathrm{MHz}$, respectively. The control of the sample temperature was better than $\pm 1 \mathrm{~K}$. All laboratory-frame spin-lattice relaxation curves were found to be monoexponential over at least 1-2 decades. The experimental error of the relaxation times corresponds to the size of the symbols in the plots or is less.

Linear PDES (weight-average molecular weight $M_{m}=328000$; polydispersity $M_{\mathrm{r}} / M_{\mathrm{n}}=1.5$ ) was prepared by ring-opening polymerization of hexaethylcyclotrisiloxane in the presence of $\mathrm{KOH}$ as initiator. The investigated fraction was separated from oligomers by repeated precipitation in methanol. The molecular 


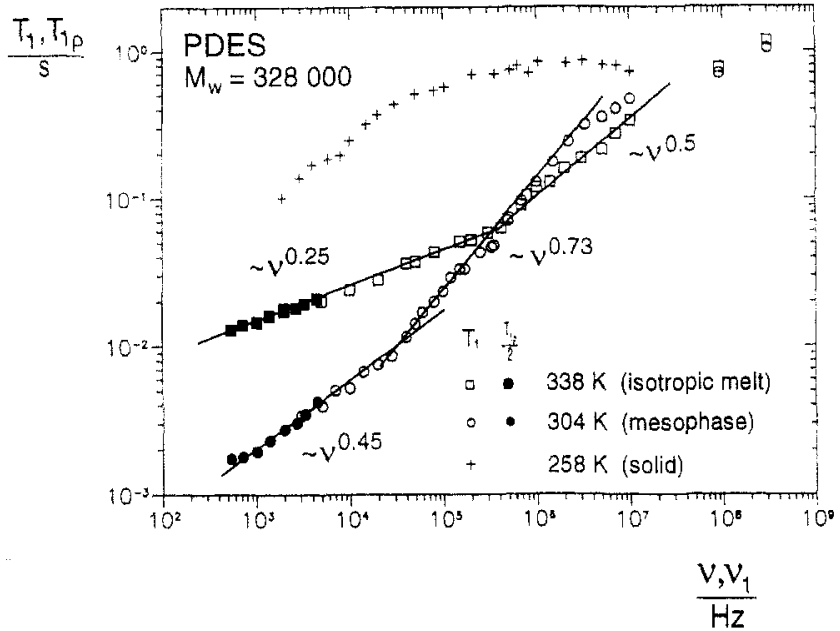

Figure 1. Frequency dependences of the proton spin-lattice relaxation times $T_{1}$ and $T_{1 p}$ in the laboratory and rotating frames of reference, respectively, in liquid and solid phases of PDES. The solid lines indicate the power laws discussed in the text.

weights were determined by GPC and osmometry. The temperature ranges of the diverse phases were determined calorimetrically. ${ }^{18}$

Linear PDMS $\left(M_{w}=250000\right)$ was purchased from Polysciences, Warrington, PA. The polydispersity was unspecified. Comparative experiments with a sample supplied by Polymer Standard Service, Mainz, Germany, with $M_{\mathrm{w}}=220000$ and $M_{\mathrm{w}} /$ $M_{\mathrm{n}}=1.2$ led to equivalent $T_{1}$ values and the same frequency dependence. ${ }^{5}$

\section{Results}

Figure 1 shows proton $T_{1}$ and $T_{1 \rho}$ dispersion data recorded in isotropic liquid, mesomorphic, and solid phases of PDES. The frequencies effective in the laboratory and rotating frames of reference are $2 \pi \nu=\gamma B_{0}$ and $2 \pi \nu_{1}=\gamma B_{1}$, respectively, where $\gamma$ is the gyromagnetic ratio, $B_{0}$ the flux density of the stationary magnetic field, and $B_{1}$ the flux density corresponding to half of the radio-frequency amplitude.

The spin-lattice relaxation data of the liquid phases can be represented for the most part by power laws section by section. Only at the highest frequencies $\left(\nu>10^{7} \mathrm{~Hz}\right)$ a slight deviation due to local segment fluctuations (i.e., rotational isomerism within the Kuhn segments, restricted rotational segment diffusion, and, in principle, side-chain motions) appears. In our previous work this sort of motion was referred to as "component $A$ " of the fluctuating dipolar interactions. 5,6 The dynamic modes of the main chain, on the other hand, that is, "component $B$ ", reveal themselves by the following power laws evaluated directly from the dispersion curves (see solid lines in Figure 1).

Isotropic melt:

$$
\begin{aligned}
& T_{1}, T_{1 \rho} \propto \\
& \left\{\begin{array}{lll}
\nu^{0.50} & \text { for } & \nu>4 \times 10^{5} \mathrm{~Hz} \text { (regime I) } \\
\nu^{0.25}, \nu_{1}^{0.25} & \text { for } & \nu, \nu_{1}<4 \times 10^{5} \mathrm{~Hz} \text { (regime II) }
\end{array}\right.
\end{aligned}
$$

Mesophase:

$$
\begin{aligned}
& T_{1}, T_{1 \rho} \propto
\end{aligned}
$$

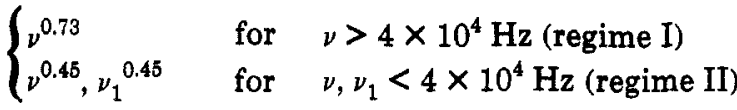

The exponents in the power laws, the reliability range of

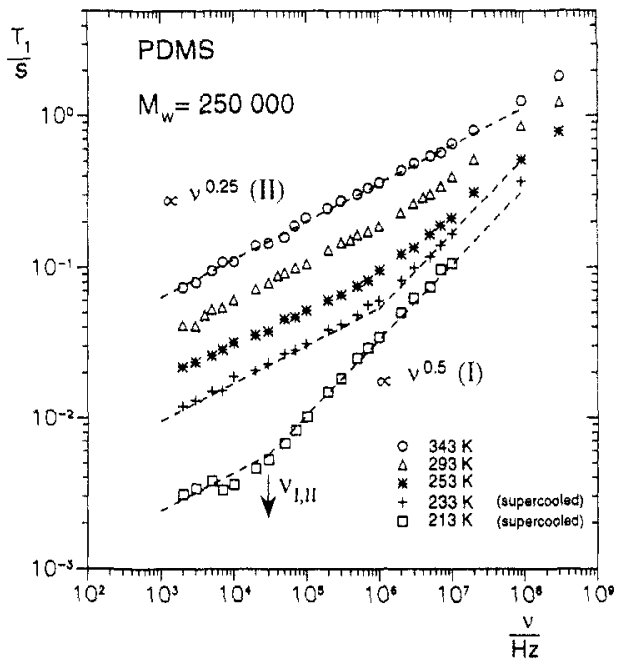

Figure 2. Frequency dependences of the proton spin-lattice relaxation time $T_{1}$ in isotropic melts of PDMS at different temperatures. The dashed lines indicate the power laws discussed in the text.

which is \pm 0.03 , and the crossover frequencies between regimes I and II are remarkably different in the two phases. In the case of the mesophase, the exponents are significantly larger. The crossover frequency (defined by the intersection of the solid lines) is shifted by a factor of 16 to lower frequencies. Roughly assuming the same activation energy of $58 \mathrm{~kJ} / \mathrm{mol}$ as for PDMS, ${ }^{5}$ one comparatively estimates a shift factor of 10 according to the different measuring temperatures of 304 and $338 \mathrm{~K}$.

The $T_{1}$ dispersion in the solid phase, on the other hand, is characterized by a tendency toward a plateau $T_{1} \approx 1 \mathrm{~s}$ at high frequencies. Correspondingly fast fluctuations of the ethyl groups with correlation times less than $10^{-9} \mathrm{~s}$ are most likely responsible for this plateau. The dispersion appearing at the low-frequency end of the scale is attributed to slow motions in uncrystallized parts or to mobile crystal defects. The main-chain relaxation efficiencies of both coexisting modifications ${ }^{18}$ of undistorted crystalline regions are considered to be minor so that no contribution in our frequency scale is expected.

The power laws describing the spin-lattice relaxation dispersion in the isotropic melt, eq 3 , are the same as those found for melts of many other polymer species and, of particular significance in this context, of PDMS (Figure 2). Merely the crossover frequency $\nu_{1, \text { II }}$ between regimes I and II is lowered by a factor of about 10 at equivalent temperatures. It is concluded that the fluctuation rates in PDES therefore must be slowed down also by a factor of 10 .

In this context it should be noted that the $T_{1}$ dispersion of melts of PDMS and many other polymers turned out to be independent of molecular weight as long as the molecular weight is far above the critical value. ${ }^{5}$ The same behavior is assumed for PDES.

\section{Discussion of the $T_{1}$ Dispersions of the PDES Mesophase Relative to Isotropic Polymer Melts in General}

Current theories on polymer dynamics taking into account the interchain excluded-volume forces (in contrast to the Rouse model ${ }^{19}$ ) are often based on the memory function formalism. ${ }^{20-22}$ Recently we have shown that such memory functions are directly related to the $T_{1}$ dispersion. ${ }^{6,17}$ In this way the apparently universal power laws eq 3 found in previous experiments and reproduced in this study can be interpreted as two dynamic limits of 
Schweizer's renormalized Rouse approach, ${ }^{22}$ for instance. The agreement with the theoretical prediction appears to be perfect.

In this context the $T_{1}$ dispersion behavior of the PDES mesophase is of particular interest: The chain conformation must mirror the ordered microstructure existing in this phase $e^{1,2}$ so that strong deviations from Gaussian random coils, which usually are assumed for isotropic melts, can be expected. The PDES mesophase may be classified as a "columnar liquid crystal" meaning that the axes of the still highly disordered chains with respect to rotational isomerism tend to be aligned within the ordered domains.

In the Doi/Edwards tube model ${ }^{19}$ for isotropic melts, on the other hand, randomly coiled chains are considered which are confined to a "tube" which itself has a randomly coiled conformation. Depending on the time and length scales considered, the time dependences of dynamic quantities such as the mean-square segment displacement are given by certain power laws, e.g., $\left\langle r_{s}{ }^{2}\right\rangle \propto t^{1 / 2}$ and $\left\langle r_{s}{ }^{2}\right\rangle$ $\propto t^{1 / 4}$.

Extrapolating this picture to the partially ordered microstructure of the PDES mesophase, one expects an entirely different segment displacement behavior. The chains tend to be aligned along a director. Compared with the isotropic melt, the conformation of the chain contour is less random. If the physical nature of the dynamic mechanisms is not altered not withstanding the conformational ordering of the chains, one expects, as a consequence, merely larger exponents in the power-law limits of the time-dependent mean-square segment displacement.

In a previous paper ${ }^{23}$ we have shown that the $T_{1}$ dispersion can reflect the limits of the mean-square segment displacement provided that the segments are displaced strictly along the chain contour in the time scale under consideration. This is certainly a limitation which must be regarded carefully but suggests the broadly correct $T_{1}$ dispersion behavior in the sense that the time dependence of the mean-square segment displacement is mirrored: The experimental spin-lattice relaxation dispersion of the mesophase can be described by power laws with exponents increased by a factor of 1.8 in regime II and a factor of 1.5 in regime I relative to the isotropic melts (eqs 4 and 3).

A less qualitative interpretation would be possible on the basis of a memory function theory. Unfortunately no such treatment of mesophases is available yet.

\section{Comparison of the $T_{1}$ Dispersions of PDES and Main-Chain Liquid-Crystal Polymers}

The $T_{1}$ dispersion behavior of the PDES mesophase has been discussed so far in the conceptual framework developed for melts of flexible polymers. Alternatively, one can refer to the dynamics of nematic main-chain liquidcrystal polymers, that is, of chains containing mesogenic groups. ${ }^{7}$

The dynamic process which is particularly characteristic of nematic phases is the fluctuation of the order director characterized by a square-root frequency dependence (eq 1). A corresponding dispersion region was resolved with diverse nematic substances ${ }^{9,24}$ including main-chain liquidcrystal polymers and their monomers. ${ }^{7}$ With polymerized materials, ${ }^{7}$ the influence of this mechanism appears, however, to be partly concealed by local (i.e., noncollective) segment fluctuations, that is, by restricted rotational diffusion or rotational isomerism. On the other hand, the two types of processes can experimentally be distinguished by the effect of isotropization: Order director fluctuations vanish with isotropization, of course, whereas local segment fluctuations should essentially remain unaffected. A further argument is that the restricted rotational diffusion process observed in monomeric liquid crystals turns out to be equivalent to the local segment fluctuations of polymeric materials. Thus the explanation of the $T_{1}$ dispersion of main-chain liquid crystal polymers appears to be well established.

This interpretation cannot, however, be straightforwardly applied to the mesophase of PDES: the observed low-frequency dispersion of the PDES mesophase (eq 4, regime II) does not rule out any influence of order fluctuations (eq 1). The difference between the exponent 0.45 found in the PDES mesophase, and the theoretical prediction of $1 / 2$ for nematic order fluctuations is not significant. On the other hand, there is no indication of local segment fluctuations in our frequency window. The $T_{1}$ dispersion of the PDES mesophase evidently changes with isotropization, so that local segment fluctuations are ruled out as a relaxation mechanism in regime $\mathrm{I}$.

We rather assume that chain modes which are strongly influenced by the characteristic microstructure of the mesophase are responsible for the $T_{1}$ dispersion in this regime. The influence of local segment fluctuations in flexible polymers (addressed above as component $A$ ) hence must be restricted to the highest frequencies of our range and reveals itself merely as a slight deviation from the indicated power laws in Figures 1 and 2. (At least with isotropic melts this interpretation can be confirmed by considering the $T_{1}$ minimum of the temperature dependence which is indicative for component A. ${ }^{6}$ )

The fundamental difference between the PDES mesophase and the nematic phase of main-chain liquid-crystal polymers may also be seen by the fact that samples consisting of the monomers of main-chain liquid-crystal polymers adopt ordered liquid phases in the same way, whereas diethylsiloxane monomers or oligomers do not. ${ }^{1}$ This comparison demonstrates that the PDES mesophase microstructure is not a consequence of interactions in segment length scales as in the case of main-chain liquidcrystal polymers.

\section{Conclusions}

The $T_{1}$ dispersions of PDES in the isotropic melt and in the mesophase are virtually characterized by two powerlaw sections in each case. In the mesophase the exponents are significantly increased and the crossover frequency between the two regimes is strongly reduced. Isotropization thus clearly affects the character of polymer dynamics. The high-frequency relaxation data of the solid material are essentially governed by side-group motions in contrast to the liquid phases.

The relaxation behavior of isotropic PDES melts evidently results from chain modes and is entirely equivalent to that of PDMS melts and isotropic melts of many other polymer species. A universal interpretation scheme based on the memory function formalism therefore can be applied as suggested in previous papers.

Molecular dynamics in the PDES mesophase as reflected by the $T_{1}$ dispersion is qualitatively different from processes occurring in nematic main-chain liquid-crystal polymers. The high-frequency $T_{1}$ dispersion of main-chain liquid-crystal polymers has been attributed to local segment fluctuations whereas that of the PDES mesophase is due to chain modes. The power law describing the lowfrequency dispersion of the PDES mesophase can also be explained by chain modes (in a different dynamic limit). The influence of collective order fluctuations which 
produce a similar relaxation behavior cannot, however, be excluded on this basis. Anyhow, the prevailing conclusion from this study, on the whole, is that the chain dynamics in the PDES mesophase mimics the chain modes in isotropic melts modified for a microstructure with reduced randomness. Remarkably, the microstructural differences affect the chain dynamics already in a time scale as short as $10^{-8} \mathrm{~s}$.

Acknowledgment. This work was supported by the Deutsche Forschungsgemeinschaft (SFB 239).

\section{References and Notes}

(1) Godovsky, Y. K.; Papkov, V. S. Makromol. Chem., Macromol. Symp. 1986, 4, 71 .

(2) Kögler, G.; Loufakis, K.; Möller, M. Polymer 1990, 31, 1538.

(3) Kimmich, R. Bull. Magn. Reson. 1980, 1, 195.

(4) Noack, F. Prog. NMR Spectrosc. 1986, 18, 171.

(5) Weber, H. W.; Kimmich, R. Macromolecules 1993, 26, 2597.

(6) Kimmich, R.; Fatkullin, N.; Weber, H. W.; Stapf, S. J. NonCrystalline Solids, in press (1994).

(7) Zeuner, U.; Dippel, T.; Noack, F.; Mûller, K.; Mayer, C.; Heaton, N.; Kothe, G. J. Chem. Phys. 1992, 97, 3794.
(8) Schweikert, K. H.; Noack, F. Mol. Cryst. Liq. Cryst. 1992, 212, 33.

(9) Köllner, R.; Schweikert, K. H.; Noack, F.; Zimmermann, H. Liq. Cryst. 1993, 13, 483.

(10) Pincus, P. Solid State Commun. 1969, 7, 415.

(11) Blinc, R.; Hogenboom, D.; O'Reilly, D.; Peterson, E. Phys. Rev. Lett. 1969, 23, 969.

(12) Doane, J. W.; Johnson, D. L. Chem. Phys. Lett. 1970, 6, 291.

(13) Ukleja, P.; Pirs, J.; Doane, J. W. Phys. Rev. A 1976, 14, 414.

(14) Dong, R. Y. Isr. J. Chem. 1983, 23, 370.

(15) Vold, R. R.; Vold, R. L. J. Chem. Phys. 1988, 88, 1443.

(16) Vilfan, M.; Kogoj, M.; Blinc, R. J. Chem. Phys. 1987, 86, 1055.

(17) Fatkullin, N.; Kimmich, R.; Weber, H. W. Phys. Rev, E 1993, 47,4600 .

(18) Kögler, G.; Hasenhindl, A.; Möller, M. Macromolecules 1989, 22,4190 .

(19) Doi, M.; Edwards, S. F. The Theory of Polymer Dynamics; Clarendon Press: Oxford, U.K., 1986.

(20) Ronca, G. J. Chem. Phys. 1983, 79, 1031.

(21) Hess, W. Macromolecules 1988, 21, 2620.

(22) Schweizer, K. S. J. Chem. Phys. 1989, 91, 5802.

(23) Kimmich, R.; Weber, H. W. J. Chem. Phys. 1993, 98, 5847.

(24) Wölfel, W.; Noack, F.; Stohrer, M. Z. Naturforsch. 1975, 30A, 437. 